PROCEEDINGS OF THE

AMERICAN MATHEMATICAL SOCIETY

Volume 135, Number 11, November 2007, Pages 3599-3606

S 0002-9939(07)08889-2

Article electronically published on June 29, 2007

\title{
TWO CLASSES OF SPECIAL FUNCTIONS USING FOURIER TRANSFORMS OF SOME FINITE CLASSES OF CLASSICAL ORTHOGONAL POLYNOMIALS
}

\author{
WOLFRAM KOEPF AND MOHAMMAD MASJED-JAMEI \\ (Communicated by Carmen C. Chicone)
}

\begin{abstract}
Some orthogonal polynomial systems are mapped onto each other by the Fourier transform. The best-known example of this type is the Hermite functions, i.e., the Hermite polynomials multiplied by $\exp \left(-x^{2} / 2\right)$, which are eigenfunctions of the Fourier transform. In this paper, we introduce two new examples of finite systems of this type and obtain their orthogonality relations. We also estimate a complicated integral and propose a conjecture for a further example of finite orthogonal sequences.
\end{abstract}

\section{INTRODUCTION}

Let us start our discussion with the generic differential equation of the classical orthogonal polynomials, i.e.,

$$
\left(a x^{2}+b x+c\right) y_{n}^{\prime \prime}(x)+(d x+e) y_{n}^{\prime}(x)-n(d+(n-1) a) y_{n}(x)=0,
$$

in which $a, b, c, d, e$ are all real parameters and $n$ is a positive integer. In general, six special classes of orthogonal polynomials can be extracted from the differential equation (11). Three of them, namely the Jacobi, Laguerre and Hermite polynomials, are known as the infinite classical orthogonal polynomials. Three other classes, which are less well known, are the finite classical orthogonal polynomials that are respectively orthogonal with respect to the generalized $T$, inverse Gamma and $F$ distributions; see [Les, Mas1, Mas2 for more details.

Since the general properties of the finite classes whose weight functions correspond to the inverse Gamma and $F$ distributions [PFTV, WF] are required in this paper, we restate them here in summary.

1.1. Finite classical orthogonal polynomials with weight $W_{1}(x, p)=x^{-p} e^{-\frac{1}{x}}$ on $[0, \infty)$. If $(a, b, c, d, e)=(1,0,0,-p+2,1)$ is chosen in (11), then the equation

$$
x^{2} y_{n}^{\prime \prime}(x)+((2-p) x+1) y_{n}^{\prime}(x)-n(n+1-p) y_{n}(x)=0
$$

Received by the editors January 1, 2006 and, in revised form, August 16, 2006.

2000 Mathematics Subject Classification. Primary 33C45.

Key words and phrases. Classical orthogonal polynomials, Fourier transform, hypergeometric functions, Gosper identity, Ramanujan integral. 
has the explicit polynomial solution Mas2

$$
N_{n}^{(p)}(x)=(-1)^{n} \sum_{k=0}^{n} k !\left(\begin{array}{c}
p-(n+1) \\
k
\end{array}\right)\left(\begin{array}{c}
n \\
n-k
\end{array}\right)(-x)^{k} .
$$

It was shown in Mas2] that the finite set $\left\{N_{n}^{(p)}(x)\right\}_{n=0}^{N}$ is orthogonal with respect to the weight function $W_{1}(x, p)=x^{-p} e^{-\frac{1}{x}}$ on $[0, \infty)$ if and only if $p>2 N+1$. The orthogonality relation corresponding to these polynomials is given by

$$
\begin{aligned}
& \int_{0}^{\infty} x^{-p} e^{-\frac{1}{x}} N_{n}^{(p)}(x) N_{m}^{(p)}(x) d x=\left(\frac{n !(p-(n+1)) !}{p-(2 n+1)}\right) \delta_{n, m} \\
& \quad \text { for } m, n=0,1,2, \ldots, N<\frac{p-1}{2},
\end{aligned}
$$

where $\delta_{n, m}= \begin{cases}0 & \text { if } n \neq m \\ 1 & \text { if } n=m\end{cases}$

1.2. Finite classical orthogonal polynomials with weight $W_{2}(x, p, q)=$ $\frac{x^{q}}{(1+x)^{p+q}}$ on $[0, \infty)$. Similarly, if $(a, b, c, d, e)=(1,1,0,-p+2, q+1)$ is considered in (11), then the equation

$$
x(x+1) y_{n}^{\prime \prime}(x)+((2-p) x+q+1) y_{n}^{\prime}(x)-n(n+1-p) y_{n}(x)=0
$$

has the polynomial solution Mas2

$$
M_{n}^{(p, q)}(x)=(-1)^{n} n ! \sum_{k=0}^{n}\left(\begin{array}{c}
p-(n+1) \\
k
\end{array}\right)\left(\begin{array}{l}
q+n \\
n-k
\end{array}\right)(-x)^{k} .
$$

According to Mas2], it can be shown that the finite set $\left\{M_{n}^{(p, q)}(x)\right\}_{n=0}^{N}$ is orthogonal with respect to the weight function $W_{2}(x, p, q)=\frac{x^{q}}{(1+x)^{p+q}}$ on $[0, \infty)$ if and only if $q>-1$ and $p>2 N+1$. The corresponding orthogonality relation takes the form $(7)$

$$
\int_{0}^{\infty} \frac{x^{q}}{(1+x)^{p+q}} M_{n}^{(p, q)}(x) M_{m}^{(p, q)}(x) d x=\left(\frac{n !(p-(n+1)) !(q+n) !}{(p-(2 n+1))(p+q-(n+1)) !}\right) \delta_{n, m}
$$

for $m, n=0,1,2, \ldots, N<\frac{p-1}{2}, q>-1$.

Some orthogonal polynomial systems are mapped onto each other by the Fourier transform or by another integral transform such as the Mellin and Hankel transforms (for integral transforms, see [EMOT]). The best-known examples of this type are the Hermite functions, i.e. the Hermite polynomials $H_{n}(x)$ multiplied by $\exp \left(-x^{2} / 2\right)$, which are eigenfunctions of the Fourier transform. For more examples we refer the reader to [Koor1, Koor2] and Koel]. The latter showed that the Jacobi and continuous Hahn polynomials can be mapped onto each other in such a way, and the orthogonality relations for the continuous Hahn polynomials then follow from the orthogonality relations for the Jacobi polynomials and the Parseval formula. 


\section{Fourier transform of TWO Finite ClASSES OF ORTHOGONAL POLYNOMIALS AND THEIR ORTHOGONALITY PROPERTIES}

To derive the Fourier transform of the polynomials $M_{n}^{(p, q)}(x)$ and $N_{n}^{(p)}(x)$ defined in (3) and (6), we will use the well-known identity for the Beta integral

$$
B(a, b)=\int_{0}^{1} x^{a-1}(1-x)^{b-1} d x=\int_{0}^{\infty} \frac{t^{a-1}}{(1+t)^{a+b}} d t=\frac{\Gamma(a) \Gamma(b)}{\Gamma(a+b)},
$$

where

$$
\Gamma(z)=\int_{0}^{\infty} x^{z-1} e^{-x} d x, \quad \operatorname{Re}(z)>0
$$

denotes the Gamma function satisfying the fundamental recurrence relation $\Gamma(z+1)=z \Gamma(z)$. The Fourier transform of a function, say $g(x)$, is defined as

$$
\mathcal{F}(s)=\mathcal{F}(g(x))=\int_{-\infty}^{\infty} e^{-i s x} g(x) d x,
$$

and for the inverse transform one gets the formula

$$
g(x)=\frac{1}{2 \pi} \int_{-\infty}^{\infty} e^{i s x} \mathcal{F}(s) d s .
$$

The Parseval identity of Fourier theory is given by the statement

$$
\int_{-\infty}^{\infty} g(x) h(x) d x=\frac{1}{2 \pi} \int_{-\infty}^{\infty} \mathcal{F}(g(x)) \overline{\mathcal{F}(h(x))} d s
$$

for $g, h \in L^{2}(\mathbb{R})$. Now we can define the following specific functions:

$$
g(x)=\frac{e^{q x}}{\left(1+e^{x}\right)^{p+q}} M_{n}^{(r, u)}\left(e^{x}\right), \quad h(x)=\frac{e^{a x}}{\left(1+e^{x}\right)^{a+b}} M_{m}^{(c, d)}\left(e^{x}\right),
$$

in terms of $M_{n}^{(p, q)}(x)$ given by (6), to which we will apply the Fourier transform. Clearly for both defined functions the Fourier transform exists. For the function $g(x)$ defined in (12) we get

$$
\begin{aligned}
\mathcal{F}(g(x)) & =\int_{-\infty}^{\infty} e^{-i s x} \frac{e^{q x}}{\left(1+e^{x}\right)^{p+q}} M_{n}^{(r, u)}\left(e^{x}\right) d x=\int_{0}^{\infty} t^{-i s-1} \frac{t^{q}}{(1+t)^{p+q}} M_{n}^{(r, \mu)}(t) d t \\
& =(-1)^{n} n !\left(\begin{array}{c}
u+n \\
n
\end{array}\right) \sum_{k=0}^{n} \frac{(-1)^{k}(-n)_{k}(n+1-r)_{k}}{(u+1)_{k} k !}\left(\int_{0}^{\infty} \frac{t^{q-i s-1+k}}{(1+t)^{p+q}} d t\right) \\
& =(-1)^{n} \frac{(u+n) !}{u !} \sum_{k=0}^{n} \frac{(-1)^{k}(-n)_{k}(n+1-r)_{k}}{(u+1)_{k} k !} \frac{\Gamma(q-i s+k) \Gamma(p+i s-k)}{\Gamma(p+q)} \\
& =\frac{(-1)^{n} \Gamma(u+n+1) \Gamma(q-i s) \Gamma(p+i s)}{\Gamma(u+1) \Gamma(p+q)}{ }_{3} F_{2}\left(\begin{array}{c}
-n, n+1-r, q-i s \\
u+1,-p+1-i s
\end{array} \mid 1\right)
\end{aligned}
$$

where ${ }_{3} F_{2}(\cdots)$ is a special case of the hypergeometric function Koep given by

$$
{ }_{p} F_{q}\left(\begin{array}{c}
a_{1}, a_{2}, \ldots, a_{p} \\
b_{1}, b_{2}, \ldots, b_{q}
\end{array} \mid x\right)=\sum_{k=0}^{\infty} \frac{\left(a_{1}\right)_{k}\left(a_{2}\right)_{k} \cdots\left(a_{p}\right)_{k}}{\left(b_{1}\right)_{k}\left(b_{2}\right)_{k} \cdots\left(b_{q}\right)_{k}} \frac{x^{k}}{k !}
$$


$(r)_{k}=r(r+1) \cdots(r+k-1)$ denoting the Pochhammer symbol. Note that to derive (13) we have moreover used the following identities:

$$
\Gamma(a+k)=\Gamma(a)(a)_{k} \quad \text { and } \quad \Gamma(a-k)=\frac{(-1)^{k} \Gamma(a)}{(1-a)_{k}} .
$$

Now by substituting (13) in Parseval's identity we get

$$
\begin{aligned}
& 2 \pi \int_{-\infty}^{\infty} \frac{e^{(q+a) x}}{\left(1+e^{x}\right)^{(p+q+a+b)}} M_{n}^{(r, u)}\left(e^{x}\right) M_{m}^{(c, d)}\left(e^{x}\right) d x \\
& =2 \pi \int_{0}^{\infty} \frac{t^{q+a-1}}{(1+t)^{p+q+a+b}} M_{n}^{(r, u)}(t) M_{m}^{(c, d)}(t) d t \\
& =\frac{(-1)^{n+m} \Gamma(u+n+1) \Gamma(d+m+1)}{\Gamma(u+1) \Gamma(p+q) \Gamma(d+1) \Gamma(a+b)} \\
& \times \int_{-\infty}^{\infty} \Gamma(q-i s) \Gamma(p+i s) \overline{\Gamma(a-i s) \Gamma(b+i s)}{ }_{3} F_{2}\left(\begin{array}{c}
-n, n+1-r, q-i s \\
u+1,-p+1-i s
\end{array} \mid 1\right) \\
& \times \overline{{ }_{3} F_{2}\left(\begin{array}{c}
-m, m+1-c, a-i s \\
d+1,-b+1-i s
\end{array} \mid 1\right)} d s .
\end{aligned}
$$

On the other hand, if in the left-hand side of (16) we take

$$
u=d=q+a-1 \quad \text { and } \quad r=c=p+b+1,
$$

then according to the orthogonality relation (7), Equation (16) reads as

$$
\begin{aligned}
& \frac{(2 \pi) n !(p+b-n) !(q+a-1+n) !}{(p+b-2 n)(p+q+a+b-n-1) !} \frac{\Gamma^{2}(q+a) \Gamma(p+q) \Gamma(a+b)}{(-1)^{n+m} \Gamma(q+a+n) \Gamma(q+a+m)} \delta_{n, m} \\
& =\int_{-\infty}^{\infty} \Gamma(q-i s) \Gamma(p+i s) \overline{\Gamma(a-i s) \Gamma(b+i s)}{ }_{3} F_{2}\left(\begin{array}{c}
-n, n-p-b, q-i s \\
q+a,-p+1-i s
\end{array} \mid 1\right) \\
& \times \overline{{ }_{3} F_{2}\left(\begin{array}{c}
-m, m-p-b, a-i s \\
q+a,-b+1-i s
\end{array} \mid 1\right)} d s .
\end{aligned}
$$

This gives

Theorem 1. The special function

$$
A_{n}(x ; a, b, c, d)=\frac{\Gamma(a+d+n)}{\Gamma(a+d)}{ }_{3} F_{2}\left(\begin{array}{c}
-n, n-b-c, d-x \\
a+d,-c+1-x
\end{array} \mid 1\right)
$$

has a finite orthogonality relation of the form

$$
\begin{gathered}
\frac{1}{2 \pi} \int_{-\infty}^{\infty} \Gamma(a+i x) \Gamma(b-i x) \Gamma(c+i x) \Gamma(d-i x) A_{n}(i x ; a, b, c, d) A_{m}(-i x ; d, c, b, a) d x \\
=\frac{n !}{(b+c-2 n)} \frac{\Gamma(a+d+n) \Gamma(b+c+1-n) \Gamma(c+d) \Gamma(a+b)}{\Gamma(a+b+c+d-n)} \delta_{n, m},
\end{gathered}
$$

where $a+d>-n, b+c>2 n, a+b>0$ and $c+d>0$.

Remark 1. (i) The case $n=m=0$ of the above theorem is Barnes' first lemma from 1908; see, e.g., Bailey Bail] or Whittaker and Watson [WW] for the original proof by Barnes. 
(ii) The weight function of the orthogonality relation (20) is positive for $a=$ $d, b=c$ or $a=b, c=d$.

(iii) Note that Atakishiyev and Suslov $\mathrm{AS}$ and also Askey in Askey1 have shown that the polynomials ${ }_{3} F_{2}\left(\begin{array}{c}-n, n+a+b+c+d-1, a+i x \\ a+b, a+d\end{array} \mid \begin{array}{c}1 \\ a+k n o w n\end{array}\right.$ nowadays as Hahn polynomials) are orthogonal with respect to the weight function of the orthogonality relation (20) on $(-\infty, \infty)$ (see also Askey2), while we have proved that a rational sequence of orthogonal functions such as (19) has this property.

The mentioned approach can similarly be applied to the finite orthogonal polynomials $N_{n}^{(p)}(x)$ defined in (3). In this case, we define the specific functions

$$
u(x)=\exp \left(-p x-\frac{1}{2} e^{-x}\right) N_{n}^{(q)}\left(e^{x}\right) \text { and } \nu(x)=\exp \left(-r x-\frac{1}{2} e^{-x}\right) N_{m}^{(u)}\left(e^{x}\right) .
$$

If we then take the Fourier transform for $u(x)$, we get

$$
\begin{aligned}
\mathcal{F}(u(x)) & =\int_{-\infty}^{\infty} e^{-i s x} e^{-\left(p x+\frac{1}{2} e^{-x}\right)} N_{n}^{(q)}\left(e^{x}\right) d x=\int_{0}^{\infty} t^{-i s-1-p} e^{-\frac{1}{2 t}} N_{n}^{(q)}(t) d t \\
& =(-1)^{n} n !(q-n-1) ! \sum_{k=0}^{n} \frac{(-1)^{k}}{(q-n-1-k) ! k !(n-k) !}\left(\int_{0}^{\infty} t^{-i s-1-p+k} e^{-\frac{1}{2 t}} d t\right) \\
& =(-1)^{n} 2^{p+i s} \Gamma(p+i s) \sum_{k=0}^{n} \frac{(-n)_{k}(n+1-q)_{k}}{(1-p-i s)_{k}} \frac{\left(2^{-1}\right)^{k}}{k !} \\
& =(-1)^{n} 2^{p+i s} \Gamma(p+i s)_{2} F_{1}\left(\begin{array}{c}
-n, n+1-q \\
1-p-i s
\end{array} \mid \frac{1}{2}\right) .
\end{aligned}
$$

In this computation the following definite integral was used:

$$
\int_{0}^{\infty} t^{-i s-1-p+k} e^{-\frac{1}{2 t}} d t=2^{p+i s-k} \Gamma(p+i s-k) .
$$

Now, according to definition (21) we use Parseval's identity again and get

$$
\begin{aligned}
& 2 \pi \int_{-\infty}^{\infty} e^{-(p+r) x} e^{-e^{-x}} N_{n}^{(q)}\left(e^{x}\right) N_{m}^{(u)}\left(e^{x}\right) d x \\
& =2 \pi \int_{0}^{\infty} t^{-(p+r+1)} e^{-\frac{1}{t}} N_{n}^{(q)}(t) N_{m}^{(u)}(t) d t \\
& =(-1)^{n+m} 2^{p+r} \int_{-\infty}^{\infty} \Gamma(p+i s) \overline{\Gamma(r+i s)}
\end{aligned}
$$

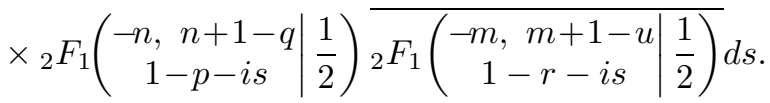

Then by assuming

$$
q=u=p+r+1
$$

and noting the orthogonality relation (4) we get the following theorem.

Theorem 2. The special function

$$
B_{n}(x ; a, b)={ }_{2} F_{1}\left(\begin{array}{c|c}
-n, n-a-b & \frac{1}{2} \\
-a+1-x & 2
\end{array}\right)
$$


has a finite orthogonality relation as follows:

(27)

$$
\frac{1}{2 \pi} \int_{-\infty}^{\infty} \Gamma(a+i x) \Gamma(b-i x) B_{n}(i x ; a, b) B_{m}(-i x ; b, a) d x=\frac{n ! \Gamma(a+b+1-n)}{(a+b-2 n) 2^{a+b}} \delta_{n, m}
$$

if $a+b>2 n$.

Remark 2. (i) If we put $n=m=0$ in (27), then we obtain

$$
\frac{1}{2 \pi} \int_{-\infty}^{\infty} \Gamma(a+i x) \Gamma(b-i x) d x=\frac{\Gamma(a+b)}{2^{a+b}} .
$$

(ii) The weight function of (27) is positive if $a=b$.

\section{Evaluating a COMPlicated integral AND A CONJECture}

By applying the Ramanujan integral Rama

$$
\begin{aligned}
\int_{-\infty}^{\infty} & \frac{d x}{\Gamma(a+x) \Gamma(b+x) \Gamma(c-x) \Gamma(d-x)} \\
& =\frac{\Gamma(a+b+c+d-3)}{\Gamma(a+c-1) \Gamma(a+d-1) \Gamma(b+c-1) \Gamma(b+d-1)}
\end{aligned}
$$

one can obtain the explicit value of the following definite integral:

$$
\begin{aligned}
I_{n}(m)= & \int_{-\infty}^{\infty}{ }_{3} F_{2}\left(\begin{array}{cc}
-n, & n-b-c, a-x \\
a+d, & -c+1-x
\end{array} \mid 1\right) \frac{(d-x)_{m}}{(1-b-x)_{m}} \\
& \times \frac{d x}{\Gamma(1-a+x) \Gamma(1-d+x) \Gamma(1-c-x) \Gamma(1-b-x)} .
\end{aligned}
$$

We write

$$
\begin{aligned}
& I_{n}(m)=\sum_{k=0}^{n} \frac{(-n)_{k}(n-b-c)_{k}}{(a+d)_{k} k !} \\
& \quad \times \int_{-\infty}^{\infty} \frac{(a-x)_{k}(d-x)_{m}}{(1-c-x)_{k}(1-b-x)_{m}} \frac{d x}{\Gamma(1-a+x) \Gamma(1-d+x) \Gamma(1-c-x) \Gamma(1-b-x)},
\end{aligned}
$$

and since

$$
\frac{(a-x)_{k}}{\Gamma(1-a+x)}=\frac{(-1)^{k}}{\Gamma(1-a+x-k)} \text { and } \frac{(d-x)_{m}}{\Gamma(1-d+x)}=\frac{(-1)^{m}}{\Gamma(1-d+x-m)},
$$

according to Ramanujan's integral defined in (29) $I_{n}(m)$ can be simplified towards

$$
\begin{aligned}
I_{n}(m)= & \sum_{k=0}^{n} \frac{(-n)_{k}(n-b-c)_{k}}{(a+d)_{k} k !}(-1)^{k+m} \\
& \quad \times \int_{-\infty}^{\infty} \frac{d x}{\Gamma(1-a+x-k) \Gamma(1-d+x-m) \Gamma(1-c-x+k) \Gamma(1-b-x+m)} \\
= & \sum_{k=0}^{n} \frac{(-1)^{k+m}(-n)_{k}(n-b-c)_{k} \Gamma(1-a-b-c-d)}{(a+d)_{k} k ! \Gamma(1-a-c) \Gamma(1-b-d) \Gamma(1-a-b+m-k) \Gamma(1-c-d-m+k)} \\
= & \frac{(-1)^{m} \Gamma(1-a-b-c-d)_{3} F_{2}\left(\begin{array}{cc}
-n, & n-b-c, a+b-m \\
a+d, & 1-c-d-m
\end{array}\right)}{\Gamma(1-a-c) \Gamma(1-b-d) \Gamma(1-a-b+m) \Gamma(1-c-d-m)} .
\end{aligned}
$$


On the other hand, the Gosper-Saalschütz identity AAR, p. 116, implies that if $e=a+b+c+1-d$, then

$$
\begin{aligned}
{ }_{3} F_{2}\left(\begin{array}{cc|}
a, & b, c \\
d, & e
\end{array} \mid 1\right)= & \frac{\pi^{2}}{\cos (d \pi) \cos (e \pi)+\cos (a \pi) \cos (b \pi) \cos (c \pi)} \\
& \times \frac{\Gamma(d)}{\Gamma(d-a) \Gamma(d-b) \Gamma(d-c)} \frac{\Gamma(e)}{\Gamma(e-a) \Gamma(e-b) \Gamma(e-c)} .
\end{aligned}
$$

Therefore the final value of the definite integral $I_{n}(m)$ is given as

$$
\begin{aligned}
& I_{n}(m)=\frac{\pi^{2} \Gamma(a+d)}{(\cos ((a+b) \pi) \cos ((b+c) \pi)-\cos ((a+d) \pi) \cos ((c+d) \pi)) \Gamma(1-a-c) \Gamma(1-b-d)} \\
& \times \frac{1}{\Gamma(a+d+n) \Gamma(a+b+c+d-n) \Gamma(d-b+m) \Gamma(1-c-d-m+n) \Gamma(1+b-d-m-n) \Gamma(1-a-b+m)} .
\end{aligned}
$$

Conjecture. We evaluated the complicated integral (30) to claim that the function $A_{n}(x ; a, b, c, d)$ defined in Theorem 1 can essentially be orthogonal with respect to the Ramanujan integral. In other words, we conjecture that

$$
\begin{gathered}
\int_{-\infty}^{\infty}{ }_{3} F_{2}\left(\begin{array}{cc}
-n, & n-b-c, d-x \\
a+d, & -b+1-x
\end{array} \mid 1\right){ }_{3} F_{2}\left(\begin{array}{cc}
-m, & m-b-c, a-x \\
a+d, & -c+1-x
\end{array} \mid 1\right) \\
\times \frac{d x}{\Gamma(1-a+x) \Gamma(1-d+x) \Gamma(1-c-x) \Gamma(1-b-x)}=K_{n} \delta_{n, m} .
\end{gathered}
$$

Again, like Remark 1(iii), the orthogonality relation of the above rational functions would complement Theorem 2.1 of the paper Askey2, which states that the polynomials

$$
q_{n}(x)=\frac{(\alpha+\gamma)_{n}(\alpha+\delta)}{n !}{ }_{3} F_{2}\left(\begin{array}{cc}
-n, & n+\alpha+\beta+\gamma+\delta-1, \\
\alpha+\gamma, & \alpha-x \\
\alpha+\delta & 1
\end{array}\right)
$$

satisfy the orthogonality relation

$$
\begin{aligned}
& \int_{-\infty}^{\infty} q_{n}(x) q_{m}(x) \times \frac{d x}{\Gamma(1-\alpha+x) \Gamma(1-\beta+x) \Gamma(1-\gamma-x) \Gamma(1-\delta-x)} \\
& =\frac{\Gamma(2-\alpha-\beta-\gamma-\delta-n)}{(1-\alpha-\beta-\gamma-\delta-2 n) \Gamma(1-\alpha-\gamma-n) \Gamma(1-\alpha-\delta-n) \Gamma(1-\beta-\gamma-n) \Gamma(1-\beta-\delta-n)} \delta_{n, m}
\end{aligned}
$$

and have a unique Ramanujan weight function with the orthogonality (36).

\section{REFERENCES}

[AAR] G. E. Andrews, R. Askey and R. Roy, Special Functions, Encyclopedia of Mathematics and its Applications 71, Cambridge University Press, Cambridge, 1999. MR.1688958 (2000g:33001)

[Askey1] R. Askey, Continuous Hahn polynomials, J. Physics A 18, 1985, L1017-L1019. MR0812420 (87d:33021)

[Askey2] R. Askey, An integral of Ramanujan and orthogonal polynomials, J. Indian Math. Soc. 51, 1987, 27-36. MR0988306 (90d:33004)

[AS] N. M. Atakishiyev and S. K. Suslov, The Hahn and Meixner polynomials of an imaginary argument and some of their applications, J. Physics A 18, 1985, 1583-1596. MR0796065 (87i:33021)

[Bail] W. N. Bailey, Generalized Hypergeometric Series, Cambridge Tracts 32, Cambridge University PFTV, 1935. Reprinted by Hafner Publishing Company, 1972. MR 0185155 $(32: 2625)$

[EMOT] A. Erdelyi, W. Magnus, F. Oberhettinger and F. G. Tricomi, Tables of Integral Transforms, Vol. 2, McGraw-Hill, 1954. MR0065685 (16:468c) 
[Koel] H. T. Koelink, On Jacobi and continuous Hahn polynomials, Proc. Amer. Math. Soc. 124, 1996, 887-898. MR1307541 (96f:33018)

[Koep] W. Koepf, Hypergeometric Summation, Braunschweig/Wiesbaden, Vieweg, 1998. MR1644447 (2000c:33002)

[Koor1] T. H. Koornwinder, Special orthogonal polynomial systems mapped onto each other by the Fourier-Jacobi transform, Polynômes Orthogonaux et Applications (C. Brezinski, A. Draux, A. P. Magnus, P. Maroni and A. Ronveaux, Eds.), Lecture Notes Math. 1171, Springer, 1985, 174-183. MR0838982 (87g:33007)

[Koor2] T. H. Koornwinder, Group theoretic interpretations of Askey's scheme of hypergeometric orthogonal polynomials, Orthogonal Polynomials and their Applications (M. Alfaro, J. S. Dehesa, F. J. Marcellan, J. L. Rubio de Francia and J. Vinuesa, Eds.), Lecture Notes Math. 1329, Springer, 1988, 46-72. MR0973421 (90b:33024)

[Les] P. Lesky, Eine Charakterisierung der klassischen kontinuierlichen, diskreten und qOrthogonalpolynome, Shaker, Aachen, 2005.

[Mas1] M. Masjed-Jamei, Classical orthogonal polynomials with weight function $\left((a x+b)^{2}+\right.$ $\left.(c x+d)^{2}\right)^{-p} \exp (q \arctan ((a x+b) /(c x+d))) ;-\infty<x<\infty$ and a generalization of $T$ and $F$ distributions, J. Integral Transforms and Special Functions 15 (2), 2004, 137-153. MR2053407 (2005b:33011)

[Mas2] M. Masjed-Jamei, Three finite classes of hypergeometric orthogonal polynomials and their application in functions approximation, J. Integral Transforms and Special Functions 13 (2), 2002, 169-190. MR1915513 (2003i:33011)

[PFTV] W. H. Press, B. P. Flannery, S. A. Teukolsky and W. T. Vetterling, Beta Function, $T$ Student Distribution and F-Distribution, Section 6.2 in Numerical Recipes in Fortran: The Art of Scientific Computing, second edition, Cambridge University Press, Cambridge, 1992, 219-223. MR.1196230 (93i:65001a)

[Rama] S. Ramanujan, A class of definite integrals, Quarterly J. Math. 48 (1920), 294-310.

$[\mathrm{WF}]$ R. E. Walpole and J. E. Freund, Mathematical Statistics, Prentice-Hall, 1980. MR0591029 (81k:62002)

[WW] E. T. Whittaker and G. N. Watson, A Course of Modern Analysis, 4th ed., Cambridge University Press, Cambridge, 1962. MR0178117 (31:2375)

Department of Mathematics, University of Kassel, Heinrich-Plett-Str. 40, D-34132 Kassel, Germany

E-mail address: koepf@mathematik.uni-kassel.de

Department of Mathematics, K. N. Toosi University of Technology, Sayed Khandan, Jolfa Av., Tehran, Iran

E-mail address: mmjamei@yahoo.com 